\title{
Multiplicative Decompositions and Frequency of Vanishing of Nonnegative Submartingales
}

\author{
Ashkan Nikeghbali ${ }^{1}$
}

Received August 21, 2005; revised September 9, 2005

\begin{abstract}
In this paper, we establish a multiplicative decomposition formula for nonnegative local martingales and use it to characterize the set of continuous local submartingales $Y$ of the form $Y=N+A$, where the measure $d A$ is carried by the set of zeros of $Y$. In particular, we shall see that in the set of all local submartingales with the same martingale part in the multiplicative decomposition, these submartingales are the smallest ones. We also study some integrability questions in the multiplicative decomposition and interpret the notion of saturated sets in the light of our results.
\end{abstract}

KEY WORDS: random times; submartingales; general theory of stochastic processes

2000 Mathematics Subject Classification: 05C38, 15A15; 05A15, $15 \mathrm{~A} 18$.

\section{INTRODUCTION}

In Ref. 12, 13 we have introduced the following family of nonnegative local submartingales which enjoy many remarkable properties, and whose definition goes back to Yor: ${ }^{(15)}$

Definition 1.1. Let $\left(X_{t}\right)$ be a nonnegative local submartingale, which decomposes as:

$$
X_{t}=N_{t}+A_{t} .
$$

We say that $\left(X_{t}\right)$ is of class $(\Sigma)$ if:

\footnotetext{
${ }^{1}$ Departement Mathematik, ETH Zurich, HG G16, Ramistrasse 101, 8092 Zurich, Switzerland. E-mail: ashkan.nikeghbali@math.ethz.ch
} 
(1) $\left(N_{t}\right)$ is a continuous local martingale, with $N_{0}=0$;

(2) $\left(A_{t}\right)$ is a continuous increasing process, with $A_{0}=0$;

(3) the measure $\left(d A_{t}\right)$ is carried by the set $\left\{t: X_{t}=0\right\}$.

If additionally, $\left(X_{t}\right)$ is of class $(D)$, we shall say that $\left(X_{t}\right)$ is of class $(\Sigma D)$.

A natural question to ask is: what is the place of the local submartingales of class $(\Sigma)$ among other nonnegative local submartingales? In particular, is there a systematic way to construct them? This paper has two aims:

- to understand better the nature of the local submartingales of class $(\Sigma)$

- to give a characterization which will provide us with further examples of such processes.

Our approach is based on the multiplicative decompositions of nonnegative local submartingales: indeed, as will be shown, the stochastic processes of class $(\Sigma)$ reveal some of their very nice properties when they are factorized (in particular, in a sense that will be made precise, these processes have more zeros than other nonnegative local submartingales).

The theory of multiplicative decompositions of semimartingales has not received much attention in the literature and is much less powerful than the additive or Doob-Meyer decompositions of semimartingales. But in the special case of interest to us, it will be the relevant decomposition to consider.

One of the very first results on multiplicative decompositions, and which is not so well known, is due to Itô and Watanabe ${ }^{(7)}$ who established a multiplicative decomposition for nonnegative supermartingales in the framework of Markov Processes: any nonnegative supermartingale $\left(Z_{t}\right)$ is factorized as:

$$
Z_{t}=Z_{t}^{(0)} Z_{t}^{(1)}
$$

with a positive local martingale $Z_{t}^{(0)}$ and a decreasing process $Z_{t}^{(1)}\left(Z_{0}^{(1)}=\right.$ 1). Later, Meyer. ${ }^{(9)}$ Meyer and Yocurp, ${ }^{(10)}$ and Azéma ${ }^{(2)}$ have studied multiplicative decompositions of nonnegative submartingales and bounded nonnegative supermartingales to find conditions under which there exists a (unique) raw increasing process $K$ which generates them. Their approach differed from that of Itô and Watanabe and was based on the then flourishing general theory of stochastic processes. The results obtained in Refs. 2, 9, 10 are very refined and too technical for our purpose so we will not discuss them here. In fact, they do not even apply very well to our setting. Let us eventually mention that it is proved in Ref. 11 that every supermartingale $\mathbb{P}\left(L>t \mid \mathcal{F}_{t}\right)$ associated with an honest time $L$ can be represented as 
$\frac{N_{t}}{\bar{N}_{t}}$, where $\bar{N}_{t}=\sup _{u \leqslant t} N_{u}$, and where $N$ is a nonnegative local martingale starting from one and vanishing at infinity. This in turn entailed some nice connections with the theory of enlargements of filtrations. Let us eventually mention the book of Ref. 8 which contains a paragraph about multiplicative decompositions (but the result of Itô and Watanabe is not mentioned there).

The main results of this paper are the following:

(1) Every continuous nonnegative local submartingale $\left(Y_{t}\right)$, with $Y_{0}=$ 0 decomposes uniquely as:

$$
Y_{t}=M_{t} C_{t}-1,
$$

where $\left(M_{t}\right)$ is a continuous nonnegative local martingale with $M_{0}=1$ and $\left(C_{t}\right)$ is a continuous increasing process, with $C_{0}=1$. Moreover, $\left(Y_{t}\right)$ is of class $(D)$ if and only if $\left(M_{t}\right)$ is a uniformly integrable martingale and

$$
\mathbb{E}\left[M_{\infty} C_{\infty}\right]<\infty .
$$

(2) A stochastic process $\left(X_{t}\right)$ is of class $(\Sigma)$ if and only if

$$
X_{t}=\frac{M_{t}}{I_{t}}-1,
$$

where $\left(M_{t}\right)$ is a continuous nonnegative local martingale with $M_{0}=1$ and $I_{t}=\inf _{u \leqslant t} M_{u}$. Moreover, $\left(X_{t}\right)$ is of class $(\Sigma D)$ if and only if $\left(M_{t}\right)$ is a uniformly integrable martingale and

$$
\mathbb{E}\left[\log \frac{1}{I_{\infty}}\right]<\infty .
$$

(3) Let $\left(M_{t}\right)$ be a continuous nonnegative local martingale with $M_{0}=$ 1 and let $\mathcal{E}(M)$ be the set of all nonnegative local submartingales with the same martingale part $M$ in their multiplicative decomposition. Then, there exists a unique local submartingale $Y^{\star}$ of class $(\Sigma)$ in $\mathcal{E}(M)$ and it is the smallest element in $\mathcal{E}(M)$ :

$$
\forall Y \in \mathcal{E}(M), \quad Y^{\star} \leqslant Y .
$$

This last property is reminiscent of a result of Azéma and Yor: the set of zeros of submartingales of class $(\Sigma D)$ is a saturated set.(4) We shall use our results to give some non trivial example of saturated sets $H$ which are not necessarily of the form $H=\left\{t: M_{t}=0\right\}$ for some martingale $M$. 


\section{MULTIPLICATIVE DECOMPOSITIONS}

We first prove the general decomposition formula (1.2), and then specialize to the case when $X$ is of class $(\Sigma)$. We end the section with a discussion about saturated sets.

\subsection{General Formulae and Integrability}

Let $\left(\Omega, \mathcal{F},\left(\mathcal{F}_{t}\right)_{t \geqslant 0}, \mathbb{P}\right)$ a filtered probability space, satisfying the usual assumptions, and let $Y$ be a continuous nonnegative local submartingale. It is in general impossible to obtain a multiplicative decomposition of the form $Y_{t}=Y_{t}^{(0)} Y_{t}^{(1)}$, with a positive local martingale $Y_{t}^{(0)}$ and an increasing process $Y_{t}^{(1)}\left(Y_{0}^{(1)}=1\right)$. Indeed, it is well known that once a nonnegative local martingale is equal to zero, then it remains null, while this is not true for nonnegative submartingales (consider for example $\left|B_{t}\right|$, the absolute value of a standard Brownian Motion). Hence, such multiplicative decompositions can hold only for strictly positive submartingales. But this is not of interest to us since the local submartingales of the class $(\Sigma)$ have usually many zeros. That's why we propose the following multiplicative decomposition.

Proposition 2.1. Let $\left(Y_{t}\right)_{t \geqslant 0}$ be a continuous nonnegative local submartingale such that $Y_{0}=0$. Consider its Doob-Meyer decomposition:

$$
Y_{t}=m_{t}+\ell_{t} .
$$

The local submartingale $\left(Y_{t}\right)_{t \geqslant 0}$ then admits the following multiplicative decomposition:

$$
Y_{t}=M_{t} C_{t}-1,
$$

where $\left(M_{t}\right)_{t \geqslant 0}$ is a continuous local martingale, which is strictly positive, with $M_{0}=1$ and where $\left(C_{t}\right)_{t \geqslant 0}$ is an increasing continuous and adapted process, with $C_{0}=1$. The decomposition is unique and the processes $C$ and $M$ are given by the explicit formulae:

$$
C_{t}=\exp \left(\int_{0}^{t} \frac{d \ell_{s}}{1+Y_{s}}\right)
$$

and

$$
\begin{aligned}
M_{t} & =\left(Y_{t}+1\right) \exp \left(-\int_{0}^{t} \frac{d \ell_{s}}{1+Y_{s}}\right) \\
& =\exp \left(\int_{0}^{t} \frac{d m_{s}}{1+Y_{s}}-\frac{1}{2} \int_{0}^{t} \frac{d\langle m\rangle_{s}}{\left(1+Y_{s}\right)^{2}}\right) .
\end{aligned}
$$


Proof. Assume first that such a representation exists; then from Itô's formula:

$$
d Y_{t}=d m_{t}+d \ell_{t}=C_{t} d M_{t}+M_{t} d C_{t}
$$

from this equality, we deduce that:

$$
d m_{t}=C_{t} d M_{t}, d \ell_{t}=M_{t} d C_{t}
$$

Multiplying the second equality by $C_{t}$ yields:

$$
d C_{t}=C_{t} \frac{d \ell_{t}}{1+Y_{t}}
$$

which is equivalent to:

$$
C_{t}=\exp \left(\int_{0}^{t} \frac{d \ell_{s}}{1+Y_{s}}\right) .
$$

This establishes (2.3) and (2.4) is an immediate consequence.To prove (2.5), we notice that:

$$
d M_{t}=M_{t} \frac{d m_{t}}{1+Y_{t}}
$$

which is equivalent to:

$$
M_{t}=\exp \left(\int_{0}^{t} \frac{d m_{s}}{1+Y_{s}}-\frac{1}{2} \int_{0}^{t} \frac{d\langle m\rangle_{s}}{\left(1+Y_{s}\right)^{2}}\right) .
$$

This completes the uniqueness part of the proof. The existence part is immediate.

One is often interested by some questions of integrability about stochastic processes. The next proposition gives some necessary and sufficient conditions on $M$ and $C$ for $Y$ to be of class $(D)$. Before stating it, we prove a general lemma.

Lemma 2.2. Let $\left(M_{t}\right)_{t \geqslant 0}$ be a nonnegative uniformly integrable martingale, with $M_{0}=1$, and let $\left(C_{t}\right)_{t \geqslant 0}$ be an increasing right continuous and adapted process such that $C_{0}=1$. Then, we have:

$$
\mathbb{E}\left(M_{\infty} C_{\infty}\right)=1+\mathbb{E}\left(\int_{0}^{\infty} M_{s} d C_{s}\right) .
$$


Proof. We have:

$$
\mathbb{E}\left(M_{\infty} C_{\infty}\right)=1+\mathbb{E}\left(M_{\infty} \int_{0}^{\infty} d C_{s}\right)=1+\mathbb{E}\left(\int_{0}^{\infty} M_{\infty} d C_{s}\right)
$$

Now, the result of the lemma follows by replacing the constant stochastic process $(s, \omega) \rightarrow M_{\infty}(\omega)$ with its optional projection $(s, \omega) \rightarrow M_{s}(\omega)$.

Proposition 2.3. Let $\left(Y_{t}\right)_{t \geqslant 0}$ be a continuous nonnegative local submartingale such that $Y_{0}=0$, and consider its multiplicative decomposition given by (2.2). Then, the following are equivalent:

(1) $\left(Y_{t}\right)_{t \geqslant 0}$ is of class $(D)$;

(2) $\left(M_{t}\right)_{t \geqslant 0}$ is a uniformly integrable martingale and

$$
\mathbb{E}\left[M_{\infty} C_{\infty}\right]<\infty
$$

(3) $\left(M_{t}\right)_{t \geqslant 0}$ is a uniformly integrable martingale and

$$
\mathbb{E}\left[\int_{0}^{\infty} M_{s} d C_{s}\right]<\infty
$$

Proof. $\quad(1) \Rightarrow(2)$. Since $Y_{t}+1=M_{t} C_{t}$ and $C_{t} \geqslant 1$, we have:

$$
M_{t}=\frac{Y_{t}+1}{C_{t}} \leqslant Y_{t}+1
$$

and as $Y$ is of class $(D)$, so is $M$. Furthermore,

$$
M_{\infty} C_{\infty}=1+Y_{\infty}
$$

is integrable and this completes the proof of (2).

$(2) \Rightarrow(1)$. We have:

$$
M_{t} C_{t}=C_{t} \mathbb{E}\left[M_{\infty} \mid \mathcal{F}_{t}\right] \leqslant \mathbb{E}\left[C_{\infty} M_{\infty} \mid \mathcal{F}_{t}\right],
$$

and consequently, if (2) is satisfied, then $Y$ is of class $(D)$.

$(2) \Leftrightarrow(3)$. This last equivalence is a consequence of Lemma 2.2.

\subsection{A Multiplicative Characterization of the Class $(\Sigma)$ and Applications}

In this subsection, we shall see that Proposition 2.1 takes a special form which characterizes the local submartingales of the class $(\Sigma)$. The characterization that follows should be compared with the multiplicative characterization of the Azéma's supermartingales associated with honest times given in Ref. 11. 
Let $X$ be a local submartingale of class $(\Sigma)$ given by (1.1). Since $\left(d A_{t}\right)$ is carried by the zeros of $X:\left\{t: X_{t}=0\right\},(2.3)$ simply writes:

$$
C_{t}=\exp \left(A_{t}\right) .
$$

In fact, we still have a better result: denoting,

$$
I_{t}=\inf _{s \leqslant t} M_{s},
$$

we can prove that:

$$
C_{t}=\frac{1}{I_{t}}
$$

Proposition 2.4. Let $\left(X_{t}=N_{t}+A_{t}, t \geqslant 0\right)$ be a nonnegative, continuous local submartingale with $X_{0}=0$. Then the following are equivalent:

(1) $\left(X_{t}, t \geqslant 0\right)$ is of class $(\Sigma)$, i.e. $\left(d A_{t}\right)$ is carried by the set: $\left\{t: X_{t}=0\right\}$.

(2) There exists a unique strictly positive, continuous local martingale $\left(M_{t}\right)$, with $M_{0}=1$, such that:

$$
X_{t}=\frac{M_{t}}{I_{t}}-1,
$$

where

$$
I_{t}=\inf _{s \leqslant t} M_{s} .
$$

The local martingale $\left(M_{t}\right)$ is given by:

$$
M_{t}=\left(1+X_{t}\right) \exp \left(-A_{t}\right) .
$$

Proof. (1) $\Rightarrow$ (2) Since $C_{t}=\exp \left(A_{t}\right), d C_{t}$ is also carried by the set $\left\{t: X_{t}=0\right\}$. We also have:

$$
C_{t}-\frac{1}{M_{t}}=\frac{X_{t}}{M_{t}} .
$$

An application of Skorokhod's reflection lemma yields:

$$
C_{t}=\sup _{s \leqslant t} \frac{1}{M_{s}},
$$

that is:

$$
C_{t}=\frac{1}{I_{t}}
$$


(2) $\Rightarrow(1)$ Let

$$
X_{t}=\frac{M_{t}}{I_{t}}-1,
$$

with $M$ a continuous and strictly positive local martingale. It is clear that $X \geqslant 0$. An application of Itô's formula further yields:

$$
X_{t}=\int_{0}^{t} \frac{d M_{s}}{I_{s}}+\log \left(\frac{1}{I_{t}}\right) .
$$

Consequently, $X$ is of class $(\Sigma)$.

Remark 2.5. In the proof of Proposition 2.4, we have proved the following fact:

$$
X_{t}=\frac{M_{t}}{I_{t}}-1
$$

is a local submartingale of the class $(\Sigma)$ and its Doob-Meyer decomposition is given by:

$$
X_{t}=\int_{0}^{t} \frac{d M_{s}}{I_{s}}+\log \left(\frac{1}{I_{t}}\right) .
$$

The above proposition underlines the fact that the local submartingales of class $(\Sigma)$ are the smallest local submartingales with a given martingale part $M$ in their multiplicative decomposition. More precisely, let $\left(M_{t}\right)$ be a strictly positive and continuous local martingale with $M_{0}=1$ and denote $\mathcal{E}(M)$ the set of all nonnegative local submartingales with the same martingale part $M$ in the multiplicative decomposition (2.2). Then the following holds:

Corollary 2.6. Let

$$
Y^{\star}=\frac{M_{t}}{I_{t}}-1 .
$$

Then, $\left(Y_{t}^{\star}\right)$ is in $\mathcal{E}(M)$ and it is the smallest element of $\mathcal{E}(M)$ in the sense that:

$$
\forall Y \in \mathcal{E}(M), Y^{\star} \leqslant Y .
$$

Consequently, $\left(Y_{t}^{\star}\right)$ has more zeros than any other local submartingale of $\mathcal{E}(M)$. 
Proof. It suffices to note that any element $Y \in \mathcal{E}(M)$ decomposes as $Y_{t}=M_{t} C_{t}-1$. Since $Y$ must be nonnegative, we must have:

$$
M_{t} \geqslant \frac{1}{C_{t}} .
$$

But $\frac{1}{C_{t}}$ is decreasing, hence we have:

$$
\frac{1}{C_{t}} \leqslant I_{t}
$$

and this proves the Corollary.

We can restate the above corollary as:

Corollary 2.7. Let $Y$ be a nonnegative local submartingale, with $Y_{0}=$ 0 . Then, there exists a local submartingale $Y^{\star}$ of class $(\Sigma)$ such that:

$$
Y_{t}^{\star} \leqslant Y_{t} \quad \text { for all } t .
$$

This last property should be compared with a result of Ref. 13, according to which the set of zeros of a process of the class $(\Sigma D)$ is a saturated set; we shall come back on this point more precisely in the next subsection. For now, let us give some examples and some other applications.

Example 2.8. Let $\left(K_{t}\right)$ be a continuous local martingale, with $K_{0}=0$. Then $\left(\left|K_{t}\right|\right)$ is of class $(\Sigma)$. From Proposition 24, and Tanaka's formula we have:

$$
\left|K_{t}\right|=\exp \left(L_{t}\right) \exp \left(\int_{0}^{t} \frac{\operatorname{sgn}\left(K_{s}\right) d K_{s}}{1+K_{s}}-\frac{1}{2} \int_{0}^{t} \frac{d\langle K\rangle_{s}}{\left(1+K_{s}\right)^{2}}\right)-1,
$$

where $\left(L_{t}\right)$ is the local time at 0 of $K$. Similarly, we have:

$$
K_{t}^{+}=\exp \left(\frac{1}{2} L_{t}\right) \exp \left(\int_{0}^{t} \mathbf{1}_{\left(K_{s}>0\right)} \frac{d K_{s}}{1+K_{s}}-\frac{1}{2} \int_{0}^{t} \mathbf{1}_{\left(K_{s}>0\right)} \frac{d\langle K\rangle_{s}}{\left(1+K_{S}\right)^{2}}\right)-1 .
$$

Example 2.9. With the same notations as above, if we define further:

$$
\bar{K}_{t}=\sup _{s \leqslant t} K_{s}
$$

we have:

$$
\bar{K}_{t}-K_{t}=\exp \left(\bar{K}_{t}\right) \exp \left(-\int_{0}^{t} \frac{d K_{s}}{1+K_{s}}-\frac{1}{2} \int_{0}^{t} \frac{d\langle K\rangle_{s}}{\left(1+K_{s}\right)^{2}}\right)-1 .
$$


Now, let us give other applications of Proposition 2.4. The first one states that the local time at 0 of a continuous local martingale is always equal to a function of the infimum of some nonnegative local martingale. More precisely:

Corollary 2.10. Let $\left(K_{t}\right)$ be a continuous local martingale, with $K_{0}=$ 0 , and let $\left(L_{t}\right)$ be its local time at 0 . Then there exists a unique continuous and strictly positive local martingale $\left(M_{t}\right)$, with $M_{0}=1$, such that:

$$
L_{t}=\log \left(\frac{1}{I_{t}}\right) \text {, }
$$

where $I_{t}=\inf _{s \leqslant t} M_{s}$.

Proof. From Tanaka's formula, we have

$$
\left|K_{t}\right|=\int_{0}^{t} \operatorname{sgn}\left(K_{s}\right) d K_{s}+L_{t} .
$$

But since $|K|$ is of class $(\Sigma)$, from Proposition 2.4 and formula (2.8), there exists a unique continuous and strictly positive local martingale $\left(M_{t}\right)$, with $M_{0}=1$, such that:

$$
\left|K_{t}\right|=\int_{0}^{t} \frac{d M_{s}}{I_{s}}+\log \left(\frac{1}{I_{t}}\right) .
$$

Now, from the unicity of the additive decomposition, we obtain:

$$
L_{t}=\log \left(\frac{1}{I_{t}}\right) .
$$

Corollary 2.11. Let $\left(K_{t}\right)$ be a continuous local martingale, and define:

$$
\bar{K}_{t}=\sup _{s \leqslant t} K_{s}
$$

Then, there exist two nonnegative and continuous local martingales $\left(M_{t}^{(1)}\right)$ and $\left(M_{t}^{(2)}\right)$, with $M_{0}^{(1)}=M_{0}^{(2)}=1$, such that:

$$
\begin{aligned}
\left\{t: K_{t}=0\right\} & =\left\{t: M_{t}^{(1)}=I_{t}^{(1)}\right\}, \\
\left\{t: K_{t}=\bar{K}_{t}\right\} & =\left\{t: M_{t}^{(2)}=I_{t}^{(2)}\right\} .
\end{aligned}
$$


Proof. It suffices to note that $\left\{t: K_{t}=0\right\}=\left\{t:\left|K_{t}\right|=0\right\}$ and then apply Proposition 24 and formula (2.8).

Corollary 2.12. Let $L$ be an honest time. Assume that $L$ avoids stopping times, i.e. for all $\left(\mathcal{F}_{t}\right)$ stopping times $T, \mathbb{P}(L=T)=0$. If furthermore $\mathbb{P}\left(L \leqslant t \mid \mathcal{F}_{t}\right)$ is continuous (this happens in particular when all $\left(\mathcal{F}_{t}\right)$ martingales are continuous), then there exists a unique continuous and strictly positive local martingale $\left(M_{t}\right)$, with $M_{0}=1$, such that:

$$
\mathbb{P}\left(L \leqslant t \mid \mathcal{F}_{t}\right)=\frac{M_{t}}{I_{t}}-1=\int_{0}^{t} \frac{d M_{s}}{I_{s}}+\log \left(\frac{1}{I_{t}}\right) .
$$

Furthermore, we have:

$$
M_{\infty}=2 I_{\infty}
$$

Proof. The result follows from the fact that $\left(\mathbb{P}\left(L \leqslant t \mid \mathcal{F}_{t}\right)\right)$ is of class ( $\Sigma$ ) (see Ref. 1) and $\lim _{t \rightarrow \infty} \mathbb{P}\left(L \leqslant t \mid \mathcal{F}_{t}\right)=1$.

Remark 2.13. For the reader who is acquainted with the results of the general theory of stochastic processes and in particular with the theory of progressive enlargements of filtrations, it is easy to deduce from the above corollary that in fact $L$ may be represented as:

$$
L=\sup \left\{t: M_{t}=I_{t}\right\}=\inf \left\{t: M_{t}=I_{\infty}\right\} .
$$

Indeed, from the results of Ref. $1, L$ is the last zero of $\mathbb{P}\left(L \leqslant t \mid \mathcal{F}_{t}\right)$.

Now, let us give some necessary and sufficient conditions on $M$ for $X$ to be of class $(\Sigma D)$. Let $\left(X_{t}\right)$ be of class $(\Sigma)$ :

$$
X_{t}=N_{t}+A_{t}=\frac{M_{t}}{I_{t}}-1 .
$$

Let us also define

$$
U_{t}=\int_{0}^{t} \frac{d M_{s}}{M_{s}}=\int_{0}^{t} \frac{d N_{s}}{1+X_{s}} .
$$

Proposition 2.14. Let $\left(X_{t}\right)$ be of class $(\Sigma)$. Then the following properties are equivalent:

(1) $\left(X_{t}\right)$ is of class $(\Sigma D)$;

(2) $\left(M_{t}\right)$ is a uniformly integrable martingale and

$$
\mathbb{E}\left(\frac{M_{\infty}}{I_{\infty}}\right)<\infty
$$


(3) $\left(M_{t}\right)$ is a uniformly integrable martingale and

$$
\mathbb{E}\left(\log \left(\frac{1}{I_{\infty}}\right)\right)<\infty
$$

(4) $\left(M_{t}\right)$ is a uniformly integrable martingale and $\left(U_{t}\right)$ is an $L^{2}$ bounded martingale.

Proof. The equivalence between (1), (2) and (3) follows from Proposition 23 and the fact that here:

$$
M_{s} d C_{s}=M_{s} \frac{\left(-d I_{s}\right)}{I_{s}^{2}}=-\frac{d I_{s}}{I_{s}}=d\left(\log \frac{1}{I_{s}}\right) .
$$

It now suffices to show for example (3) $\Leftrightarrow(4)$. First, from Itô's formula, we note that:

$$
\log \frac{1}{M_{t}}=-\int_{0}^{t} \frac{d M_{s}}{M_{s}}+\frac{1}{2} \int_{0}^{t} \frac{d\langle M\rangle_{s}}{M_{s}^{2}} .
$$

Let us first show that (4) $\Rightarrow$ (3). From (2.9), we have:

$$
\begin{aligned}
\mathbb{E}\left[\sup _{s \leqslant t}\left|\log \frac{1}{M_{s}}\right|\right] & \leqslant \mathbb{E}\left[\sup _{s \leqslant t}\left|\int_{0}^{s} \frac{d M_{u}}{M_{u}}\right|+\frac{1}{2} \int_{0}^{t} \frac{d\langle M\rangle_{s}}{M_{s}^{2}}\right] \\
& \leqslant \mathbb{E}\left[\sup _{s \leqslant t}\left|U_{s}\right|\right]+\frac{1}{2} \mathbb{E}\left[\langle U\rangle_{t}\right] \\
& \leqslant C \mathbb{E}\left[\langle U\rangle_{t}^{1 / 2}\right]+\frac{1}{2} \mathbb{E}\left[\langle U\rangle_{t}\right] \\
& \leqslant C\left(\mathbb{E}\left[\langle U\rangle_{t}\right]\right)^{1 / 2}+\frac{1}{2} \mathbb{E}\left[\langle U\rangle_{t}\right],
\end{aligned}
$$

where the third inequality is obtained by an application of the BurkholderDavis-Gundy inequalities and the last inequality is a consequence of Jensen's inequality ( $C$ stands for a constant). This shows (3).

Now, let us prove that $(3) \Rightarrow(4)$. Again, from (2.9), and the BurkholderDavis-Gundy inequalities, we have, for any stopping time $T$ :

$$
\mathbb{E}\left[\langle U\rangle_{T}\right] \leqslant C\left(\left(\mathbb{E}\left[\langle U\rangle_{T}\right]\right)^{1 / 2}+\mathbb{E}\left[\log \frac{1}{I_{\infty}}\right]\right) .
$$

Now, we can choose a sequence of stopping times $\left(T_{n}\right)$, such that $\lim _{n \rightarrow \infty} T_{n}=+\infty$ and $\mathbb{E}\left[\langle U\rangle_{T_{n}}\right]<\infty$. Dividing both sides of the above 
equality by $\left(\mathbb{E}\left[\langle U\rangle_{T_{n}}\right]\right)^{1 / 2}$ and letting $n \rightarrow \infty$, we obtain by an application of the monotone convergence theorem that:

$$
\left(\mathbb{E}\left[\langle U\rangle_{\infty}\right]\right)^{1 / 2} \leqslant C\left(1+\frac{1}{\left(\mathbb{E}\left[\langle U\rangle_{\infty}\right]\right)^{1 / 2}} \mathbb{E}\left[\log \frac{1}{I_{\infty}}\right]\right)
$$

and this shows that

$$
\mathbb{E}\left[\langle U\rangle_{\infty}\right]<\infty
$$

Remark 2.15. In the course of the proof of the equivalence between assertions (3) and (4), we did not use the fact that $\left(M_{t}\right)$ is a uniformly integrable martingale. In fact, we have proved the following fact:

If $M_{t} \equiv \exp \left(U_{t}-\frac{1}{2}\langle U\rangle_{t}\right)$, where $\left(U_{t}\right)$ is a continuous local martingale, such that $U_{0}=0$, then we have:

$$
\mathbb{E}\left[\log \frac{1}{I_{\infty}}\right]<\infty \Leftrightarrow \mathbb{E}\left[\langle U\rangle_{\infty}\right]<\infty .
$$

One moment of attention shows that

$$
\log \frac{1}{I_{\infty}}=-\inf _{t \geqslant 0}\left(U_{t}-\frac{1}{2}\langle U\rangle_{t}\right)
$$

and hence:

$$
\mathbb{E}\left[\langle U\rangle_{\infty}\right]<\infty \Leftrightarrow \mathbb{E}\left[\inf _{t \geqslant 0}\left(U_{t}-\frac{1}{2}\langle U\rangle_{t}\right)\right]>-\infty .
$$

Remark 2.16. The property of being uniformly integrable for $M_{t}=$ $\exp \left(U_{t}-\frac{1}{2}\langle U\rangle_{t}\right)$ is not necessary, nor sufficient to have $\mathbb{E}\left[\langle U\rangle_{\infty}\right]<\infty$. Indeed, from Dubins-Schwarz theorem,

$$
U_{t}=B_{\langle U\rangle_{t}},
$$

where $B$ is a standard Brownian Motion. Hence, noting $T=\langle U\rangle_{\infty}$, it is equivalent to show that

$$
M_{t} \equiv \exp \left(B_{t \wedge T}-\frac{1}{2} t \wedge T\right)
$$

may be uniformly integrable without having $\mathbb{E}(T)<\infty$, and vice versa. 
Let $T \equiv T_{a}=\inf \left\{t: B_{t} \geqslant a\right\}$, with $a>0$; then the local martingale $\left(M_{t}\right)$ defined above is bounded and hence uniformly integrable; however, it is well known that $\mathbb{E}\left(T_{a}\right)=\infty$.

Now, conversely, let

$$
\sigma_{b} \equiv \inf \left\{t: B_{t}+b t=1\right\}
$$

with $b>0$. From Revuz and Yor $^{16}$ (Exercise 1.31, p. 335),

$$
\mathbb{E}\left(\exp \left(B_{\sigma_{b}}-\frac{1}{2} \sigma_{b}\right)\right)<1
$$

and consequently $M_{t}=\exp \left(B_{t \wedge \sigma_{b}}-\frac{1}{2} t \wedge \sigma_{b}\right)$ is not uniformly integrable. Nevertheless, $\sigma_{b}$ has exponential moments Ref. (Ref. 14, exercise 1.21, p. 334).

\subsection{Saturated Sets}

The aim of this paragraph is to understand better Corollary 2.6 with the help of some results of Azéma et al. ${ }^{(3)}$ and Azema and Yor. ${ }^{(4)}$ Corollary 2.6 tells us that the local submartingales of the class $(\Sigma)$ vanish more than any other local submartingale with the same local martingale component in its multiplicative decomposition. Azéma and Yor, ${ }^{(4)}$ have obtained a similar characterization, which in some sense complements Corollary 2.6: they proved that the set of zeros of submartingales of class $(\Sigma D)$, whose terminal value is almost surely nonzero, is saturated, that is every predictable set which is at the left of such a random set is in fact contained into it. The notion of saturated set was first introduced by Meyer (see Refs. 6 and 3) and it is probably his last contribution to the general theory of stochastic processes. It was then used by Azéma and Yor ${ }^{(4)}$ in the study of the set of zeros of continuous martingales and by Yor in an attempt to prove Barlow's conjecture on Brownian filtrations (Ref. 16, and for a proof of Barlow's conjecture, see Ref. 5). First, we give the definition of a saturated set, and then we cite a theorem of Azéma and Yor. Eventually, we give our own representation of saturated sets, and then we conclude giving several nontrivial examples of such sets.

In the sequel, for simplicity, we always assume that the filtration $\left(\mathcal{F}_{t}\right)$ is such that every $\left(\mathcal{F}_{t}\right)$ martingale is continuous. In particular, the predictable and optional sigma fields are equal. We assume we are given a predictable set $H$ whose end

$$
g=\sup \{t:(t, \omega) \in H\}
$$


is almost surely finite. We shall also say that a predictable random set $E$ is at the left of $g$ (or $H$ ) if the end $L$ of $E$ satisfies: $L \leqslant g$. There are several equivalent definitions of saturated sets in the literature which we have collected below (see Ref. 6 p. 135, Refs. 3,4, and 16, p. 108):

Definition 2.17. The following definitions of saturated sets are equivalent.

(1) (Ref. 16, p. 108) The predictable set $H$ is said to be saturated if for every end $L$ of a predictable set $L \leqslant g$ one has:

$$
(L(\omega), \omega) \in H .
$$

(2) (Ref. 4) The predictable set $H$ is saturated if every predictable set which is at the left of $H$ is in fact contained in $H$.

(3) (Ref. 6, p. 135) The predictable set $H$ is saturated if

$$
H=\left\{t: \mathbb{P}\left(g \leqslant t \mid \mathcal{F}_{t}\right)=0\right\},
$$

or in the very imaged terminology of Strasbourg, $H$ is equal to its predictable shadow.

Assume that $g$ avoids $\left(\mathcal{F}_{t}\right)$ stopping times, which is equivalent (with our assumptions on $\left.\left(\mathcal{F}_{t}\right)\right)$ to the fact that $\left(\mathbb{P}\left(g \leqslant t \mid \mathcal{F}_{t}\right)\right)$ is continuous. Consequently, from well known results by Azéma, $\left(\mathbb{P}\left(g \leqslant t \mid \mathcal{F}_{t}\right)\right)$ is of class $(\Sigma D)$. Now we can state the result of Azéma and Yor which complements our characterization of local submartingales of class $(\Sigma)$.

Proposition 2.18 (Azéma and Yor). ${ }^{(4)}$ Let $\left(X_{t}\right)$ be submartingale of class $(\Sigma D)$, such that

$$
\mathbb{P}\left(X_{\infty}=0\right)=0 .
$$

Define

$$
H=\left\{t: X_{t}=0\right\} .
$$

Then $H$ is a saturated set.

Remark 2.19. The characterization of Corollary 2.6 on the frequency of vanishing of the local submartingales of class $(\Sigma)$ did not require integrability and limit conditions. In the above proposition, the integrability condition is essential. Indeed, take

$$
X_{t}=\left|B_{t \wedge T_{1}}\right|,
$$


where $T_{1}=\inf \left\{t: B_{t}=1\right\}$. Here

$$
H=\left\{t \leqslant T_{1}: B_{t}=0\right\} .
$$

Now let us consider

$$
L=\sup \left\{t \leqslant T_{1}: B_{t}=I_{t}\right\},
$$

where as usual $I_{t}=\inf _{u \leqslant t} B_{u}$. Then

$$
X_{L}=\left|B_{L}\right|>0,
$$

and

$$
L \leqslant g,
$$

where $g=\sup \left\{t \leqslant T_{1}: B_{t}=0\right\}$. Consequently, $H$ is not saturated.

Now, let us give some other characterizations for saturated sets.

Proposition 2.20. Let $H$ be a saturated set whose end $g$ avoids $\left(\mathcal{F}_{t}\right)$ stopping times. Then, there exist two nonnegative and continuous local martingale $\left(M_{t}\right)$ and $\left(N_{t}\right)$, with $M_{0}=1, N_{0}=1$ and $M_{\infty}=2 I_{\infty}$ (where $I_{t}=$ $\left.\inf _{s \leqslant t} M_{s}\right), N_{\infty}=0$, such that $H$ may be represented as:

$$
H=\left\{t: M_{t}=I_{t}\right\}=\left\{t: N_{t}=\bar{N}_{t}\right\},
$$

where $\bar{N}_{t}=\sup _{u \leqslant t} N_{u}$. Moreover, the local martingales $\left(M_{t}\right)$ and $\left(N_{t}\right)$ are unique.

Proof. This is a consequence of Definition 2.17 (3) and Corollary 2.12 for the part with $M$. The part with $N$ follows from the multiplicative representation of $\left(\mathbb{P}\left(g \leqslant t \mid \mathcal{F}_{t}\right)\right)$ proved in Ref. 11: there exists a (unique) continuous local martingale $N$, with $N_{0}=1$ and $N_{\infty}=0$, such that

$$
\mathbb{P}\left(g \leqslant t \mid \mathcal{F}_{t}\right)=1-\frac{N_{t}}{\bar{N}_{t}} .
$$

We end the paper with some non trivial examples of saturated sets one may encounter in martingale theory or in the theory of diffusion processes. 
Example 2.21. Let $\left(M_{t}\right)$ be a continuous and nonnegative local martingale, such that $M_{0}=x>0$ and $\lim _{t \rightarrow \infty} M_{t}=0$. Define, for $0<a \leqslant x$ :

$$
g_{a}=\sup \left\{t \geqslant 0: M_{t}=a\right\} .
$$

It is proved in Ref. 13 that:

$$
\mathbb{P}\left(g \leqslant t \mid \mathcal{F}_{t}\right)=1-\left(\frac{M_{t}}{a}\right) \wedge 1 .
$$

Consequently, the set

$$
H=\left\{t: M_{t} \geqslant a\right\}
$$

is saturated.

We already noted in Remark 2.19 that the set $\left\{t \leqslant T_{1}: B_{t}=0\right\}$ is not saturated. Now, if we apply the previous result to the martingale $M_{t}=$ $1-B_{t \wedge T_{1}}$, we obtain that the set:

$$
H=\left\{t \leqslant T_{1}: B_{t} \leqslant 0\right\}
$$

is saturated.

We can also apply the above result to $\left(R_{t}\right)$, a transient diffusion with values in $[0, \infty)$, which has $\{0\}$ as entrance boundary. Let $s$ be a scale function for $R$, which we can choose such that:

$$
s(0)=-\infty, \quad \text { and } \quad s(\infty)=0 .
$$

Then, under the law $\mathbb{P}_{x}$, for any $x>0$, the local martingale $\left(M_{t}=-s\left(R_{t}\right)\right)$ satisfies the conditions above and for $0 \leqslant x \leqslant y$, we have:

$$
\mathbb{P}_{x}\left(g_{y}>t \mid \mathcal{F}_{t}\right)=\frac{s\left(R_{t}\right)}{s(y)} \wedge 1,
$$

where

$$
g_{y}=\sup \left\{t: R_{t}=y\right\} .
$$

By Definition 2.17 (3), the set

$$
H=\left\{t: R_{t} \leqslant y\right\}
$$

is saturated. 
Example 2.22. Let $N_{t}=1-B_{t \wedge T_{1}}$. Then $\bar{N}_{t}=1-\inf _{u \leqslant t \wedge T_{1}} B_{u}=$ $1-I_{t \wedge T_{1}}$ and $N_{\infty}=0$. Consequently, from Proposition 220, the random set

$$
H=\left\{t \leqslant T_{1}: B_{t}=I_{t}\right\}
$$

is saturated.

Now, we consider again a transient diffusion $\left(R_{t}\right)$, with values in $[0, \infty)$, which has $\{0\}$ as entrance boundary. The scale function $s$ is again chosen such that:

$$
s(0)=-\infty, \quad \text { and } \quad s(\infty)=0 .
$$

Then, under the law $\mathbf{P}_{x}$, for any $x>0$, the local martingale $\left(N_{t}=\frac{s\left(R_{t}\right)}{s(x)}\right.$, $t \geqslant 0)$ satisfies the conditions of Proposition 2.20, and we have:

$$
\mathbf{P}_{x}\left(g>t \mid \mathcal{F}_{t}\right)=\frac{s\left(R_{t}\right)}{s\left(I_{t}\right)}
$$

where

$$
g=\sup \left\{t: R_{t}=I_{t}\right\},
$$

and

$$
I_{t}=\inf _{s \leqslant t} R_{s} .
$$

Hence, the set

$$
H=\left\{t \geqslant 0: R_{t}=I_{t}\right\}
$$

is saturated.

\section{ACKNOWLEDGMENTS}

I am deeply indebted to Marc Yor whose ideas and help were essential for the development of this paper: he shared very kindly with me some unpublished notes about saturated sets. 


\section{REFERENCES}

1. Azéma, J. (1972).Quelques applications de la théorie générale des processus I. Invent. Math. 18, 293-336.

2. Azéma, J. (1978). Représentation d'une surmartingale bornée, $Z W, 45,191-212$.

3. Azéma, J., Meyer, P.A., and Yor, M. (1992). Martingales relatives. Sém.Proba. XXVI, Lecture Notes in Mathematics Vol. 1526, Springer, pp.307-321.

4. Azéma, J., and Yor, M. (1992). Sur les zéros des martingales continues, Sém.Proba. XXVI, Lecture Notes in Mathematics Vol. 1526, Springer, pp.248-306.

5. Barlow, M.T., Emery, M., Knight, F.B., Song, S., and Yor, M. (1998). Autour d'un théorème de Tsirelson sur des filtrations browniennes et non browniennes, Sém.Proba. XXXII, Lecture Notes in Mathematics Vol. 1686, Springer, pp.264-305.

6. Dellacherie, C., Maisonneuve, B., and Meyer, P.A. (1992). Probabilités et Potentiel, Chapitres XVII-XXIV: Processus de Markov (fin), Compléments de Calcul Stochastique, Hermann Paris.

7. Itô, K., and Watanabe, S. (1965). Transformation of Markov processes by multiplicative functionals. Ann. Inst. Fourier. Grenoble. 15(1), 13-30.

8. Jacod, J. (1979).Calcul stochastique et problèmes de martingales, Lecture Notes in Mathematics Vol. 714, Springer pp. 240-249.

9. Meyer, P.A. (1979). Représentations multiplicatives de sousmartingales d'après Azéma, Sém.Proba. XIII, Lecture Notes in Mathematics, Vol. 721, Springer.

10. Meyer, P.A. and Yoeurp, C. (1979). Sur la décomposition multiplicative des sousmartingaless positives, Sém.Proba. XIII, Lecture Notes in Mathematics, Vol. 721, Springer, pp.240249.

11. Nikeghbali, A., and Yor, M. (2006). Doob's maximal identity, multiplicative decompositions and enlargements of filtrations. Illinois J Math. 50(4), 791-814.

12. Nikeghbali, A. (2006). A class of remarkable martingales, Stochastic Process. Appl. 116(6), 917-938.

13. Nikeghbali, A. (2006). Enlargements of filtrations and path decompositions at non stopping times. Probab. Theory and Relat. Fields 136, 532-540.

14. Revuz, D., and Yor, M. (1999). Continuous martingales and Brownian Motion, 3rd ed. Springer, Berlin.

15. Yor, M. (1979). Les inégalités de sous-martingales comme conséquence de la relation de domination. Stochastics 3,1-15.

16. Yor, M. (1997). Some Aspects of Brownian Motion, Part II. Some Recent Martingale Problems. Birkhauser, Basel. 